\title{
Digital signature schemes with strong existential
}

\section{unforgeability [version 1; peer review: 2 approved]}

\author{
Jason Chia(D1, Ji-Jian Chin², Sook-Chin Yip1 \\ ${ }^{1}$ Faculty of Engineering, Multimedia University, Cyberjaya, Selangor, 63100, Malaysia \\ ${ }^{2}$ Faculty of Computing and Informatics, Multimedia University, Cyberjaya, Selangor, 63100, Malaysia
}

\author{
V1 First published: $16 \operatorname{Sep} 2021,10: 931$ \\ https://doi.org/10.12688/f1000research.72910.1 \\ Latest published: 16 Sep 2021, 10:931 \\ https://doi.org/10.12688/f1000research.72910.1
}

\section{Abstract}

Digital signature schemes (DSS) are ubiquitously used for public authentication in the infrastructure of the internet, in addition to their use as a cryptographic tool to construct even more sophisticated schemes such as those that are identity-based. The security of DSS is analyzed through the existential unforgeability under chosen message attack (EUF-CMA) experiment which promises unforgeability of signatures on new messages even when the attacker has access to an arbitrary set of messages and their corresponding signatures. However, the EUF-CMA model does not account for attacks such as an attacker forging a different signature on an existing message, even though the attack could be devastating in the real world and constitutes a severe breach of the security system. Nonetheless, most of the DSS are not analyzed in this security model, which possibly makes them vulnerable to such an attack. In contrast, a better security notion known as strong EUF-CMA (SEUF-CMA) is designed to be resistant to such attacks. This review aims to identify DSS in the literature that are secure in the sEUF-CMA model. In addition, the article discusses the challenges and future directions of DSS. In our review, we consider the security of existing DSS that fit our criterion in the sEUF-CMA model; our criterion is simple as we only require the DSS to be at least secure against the minimum of existential forgery. Our findings are categorized into two classes: the direct and indirect classes of sEUF-CMA. The former is inherently sEUF-CMA without any modification while the latter requires some transformation. Our comprehensive review contributes to the security and cryptographic research community by discussing the efficiency and security of DSS that are sEUF-CMA, which aids in selecting robust DSS in future design considerations.

\section{Keywords}

Cryptography, Digital Signatures, Strong Existential Unforgeability

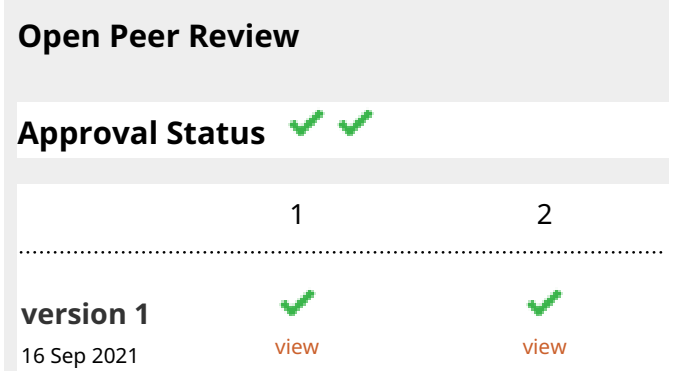

1. Denis Chee-Keong Wong, Universiti Tunku

Abdul Rahman, Kampar, Malaysia

2. Pin Shen Teh, Manchester Metropolitan

University, Manchester, UK

Any reports and responses or comments on the article can be found at the end of the article. 


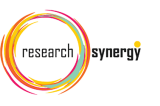

sing

This article is included in the Research Synergy

Foundation gateway.

Corresponding authors: Jason Chia (1161300548@student.mmu.edu.my), Ji-Jian Chin (ji-jian.chin@plymouth.ac.uk), Sook-Chin Yip ( scyip@mmu.edu.my)

Author roles: Chia J: Conceptualization, Data Curation, Formal Analysis, Investigation, Methodology, Software, Visualization, Writing Original Draft Preparation, Writing - Review \& Editing; Chin Jj: Conceptualization, Funding Acquisition, Methodology, Project Administration, Resources, Supervision, Validation, Writing - Review \& Editing; Yip SC: Methodology, Supervision, Validation, Writing Review \& Editing

Competing interests: No competing interests were disclosed.

Grant information: This research is supported by the Ministry of Higher Education of Malaysia through the Fundamental Research Grant Scheme under Grant FRGS/1/2019/ICT04/MMU/02/5 and in part by Multimedia University's Research Management Fund. The funders had no role in study design, data collection and analysis, decision to publish, or preparation of the manuscript.

Copyright: ( $\subset 2021$ Chia J et al. This is an open access article distributed under the terms of the Creative Commons Attribution License, which permits unrestricted use, distribution, and reproduction in any medium, provided the original work is properly cited.

How to cite this article: Chia J, Chin JJ and Yip SC. Digital signature schemes with strong existential unforgeability [version 1; peer review: 2 approved] F1000Research 2021, 10:931 https://doi.org/10.12688/f1000research.72910.1

First published: 16 Sep 2021, 10:931 https://doi.org/10.12688/f1000research.72910.1 


\section{Introduction}

The idea of a digital signature scheme (DSS) was proposed by Diffie and Hellman in 1976 as a necessity to design efficient authenticated electronic communications which can serve as legal evidence in the court of law. Rivest, Shamir, and Adleman realized the idea in their seminal work known as the RSA cryptosystem, ${ }^{2}$ the first of many. A DSS consists of three processes, as shown in Figure 1.

For every $(s k, p k) \leftarrow \operatorname{keygen}(1 k)$ and every $m$, verify $(\operatorname{sign}(m, s k), m, p k)=1$ must hold. A $\sigma$ on $m$ is valid if verify $(\sigma, m, p k)=1$. This is a standard (informal) definition of DSS. ${ }^{3}$ In the early 90 s, a paradigm known as hash-then-sign forms the industry standard for issuing digital signatures. ${ }^{4}$ The idea is to sign on the hash of a message, $h \leftarrow H(m)$ instead of the $m$ itself; this has a few benefits for $h$ is constant size, which leads to efficient signing on speed and a constant sized signature. Verification would then require the verifiers to first validate the signed hash, then perform hashing $h^{\prime} \leftarrow H(m)$ before finally comparing $h^{\prime}$ and $h$. Concrete examples of the hash-then-sign are discussed in. ${ }^{5-15}$

\section{Properties of DSS}

The following properties are required by $\mathrm{DSS}^{3}$ :

- Public verifiability: A signature $\sigma$ generated from a private key $s k$ can be verified using a public key $p k$. This property differentiates DSS from other integrity protection mechanisms, such as message authentication codes (MAC). A consequential result from this property is that signatures are also transferrable, meaning a party can copy $\sigma$ and $p k$ to use it to convince others that the message is authentic from the signer.

- Non repudiation: A signer cannot later deny that they have authenticated a message $m$ once the signature of $m, \sigma$ is generated and known. This is also another property that separates DSS from MAC, because the only entity that could have plausibly generated the signature in the case of DSS must possess the private key $s k$; whereas in a MAC scheme, the keys are shared. This property also implies that only the one in posession of $s k$ can generate valid signatures, which disallows forgeries.

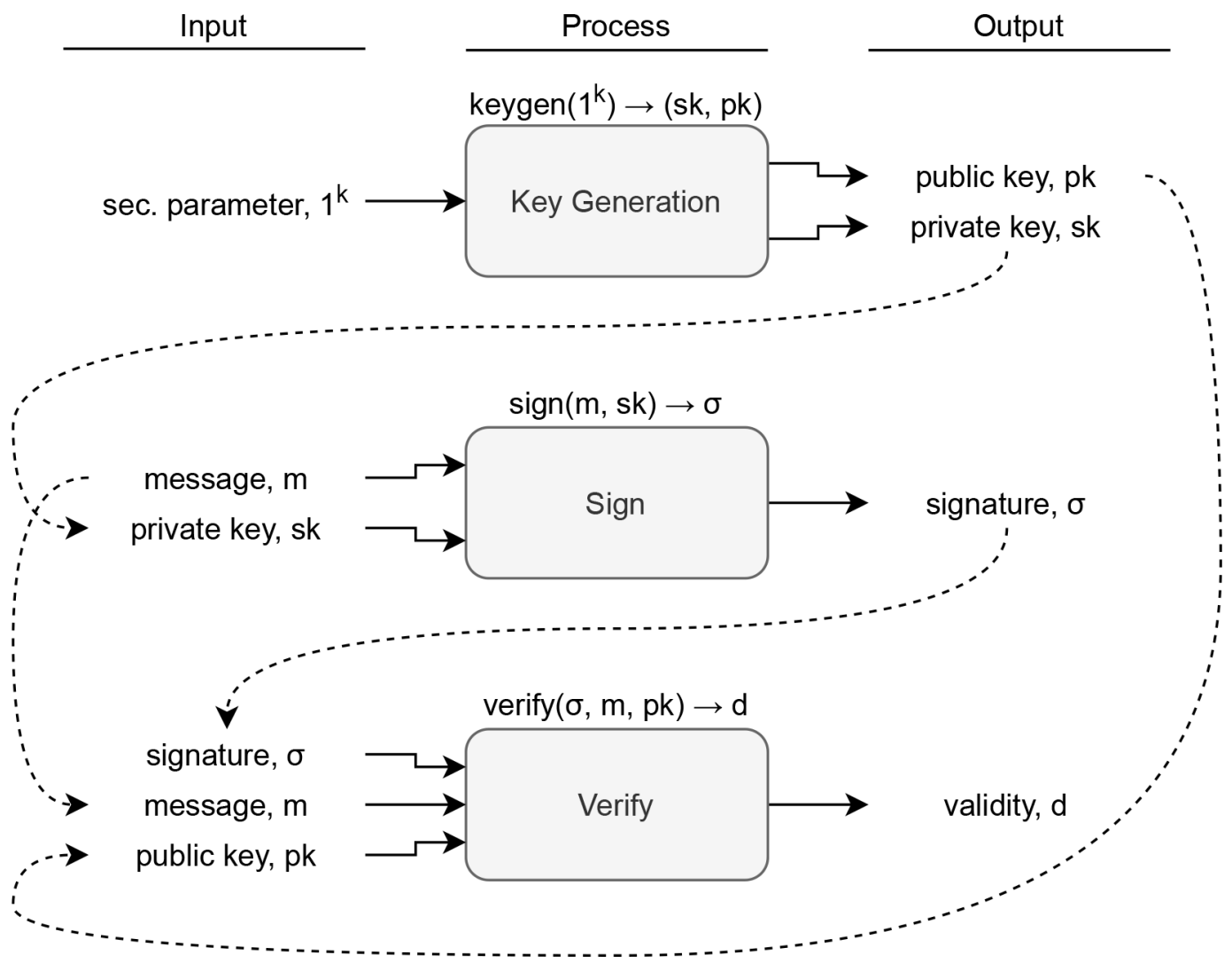

Figure 1. The three processes of a digital signature scheme and the relationship between the input/outputs. 


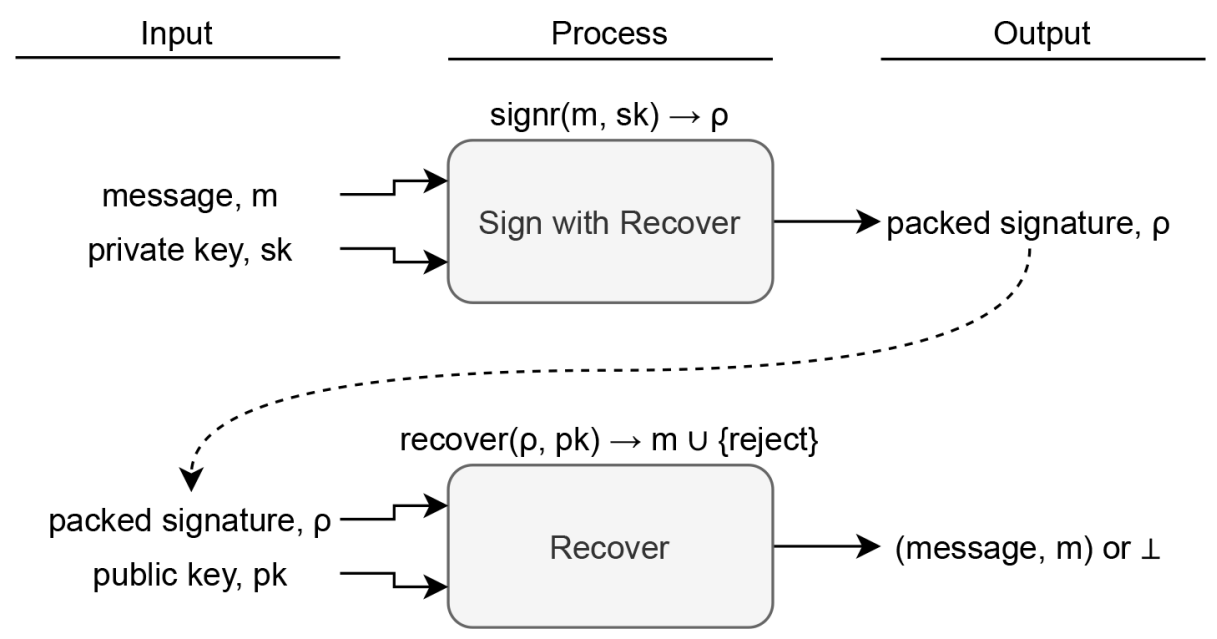

Figure 2. Sign and verify process for a digital signature scheme with message recovery.

\section{Message recovery}

Notice that the verification process requires both the message and the signature as inputs, requiring the signer to transmit both. Alternatively, some DSS can support message recovery. DSS with message recovery (DSS-R) has a different sign and verification process, shown in Figure 2; the signer only needs to transmit a packed signature $\rho$, and the verifier would recover the message $m$ successfully or abort $\perp$ depending on signature validity. For practical purposes, $|\rho| \leq|\sigma|+|m|$. Examples of DSS-R are found in previous literature. ${ }^{16-23}$

\section{Security models}

The security of DSS was first formalized by Goldwasser, Micali and Rivest in $1988 .{ }^{24}$ Different security goals are used to model the different security guarantees of a DSS when faced with an adversary. The commonly accepted goal to model against is the goal of existential forgery (EUF), which is the easiest for attackers to achieve among other goals (e.g., selective forgery, total break). In addition, ${ }^{24}$ defined the adversarial capabilities for a DSS, which aims to model what types of attacks can be carried out by an adversary when attempting to break the DSS. The strongest capability, known as adaptive chosen message (CMA) is the widely accepted adversarial capability that is the most used in the literature on DSS. Figure 3 shows the interactions of a challenger and an adversary in the EUF-CMA model. In step (1), the challenger sets up an empty set $Q$ and gives the $p k$ to the adversary. In step (2), the adversary may make oracle queries that model its chosen message attack capability. The queried messages are added to the set $Q$. In step (3), the adversary announces to the challenger the target message $m^{*}$ it wants to forge. $m^{*}$ must not be an element of $Q$, nor it can be queried to the oracle; this prevents trivial attacks which uses the oracle to break the security goal. Note, step (4) allows the adversary to use the signing oracle again, which models the adaptive nature of the attack. Finally, in step (5), the adversary outputs a forgery

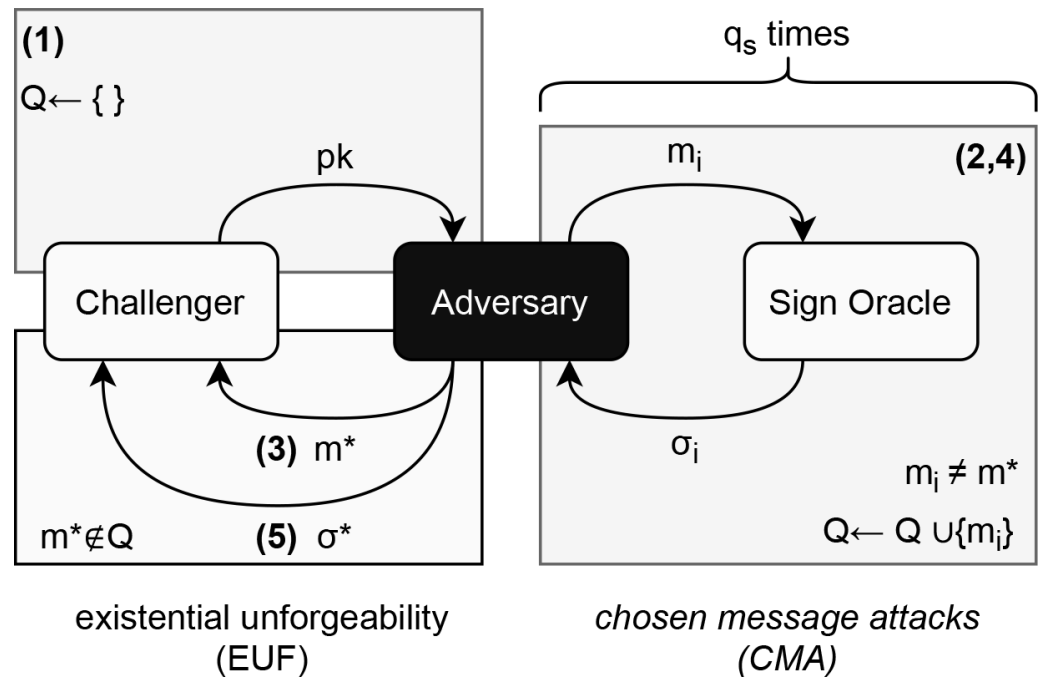

Figure 3. EUF-CMA security model. 


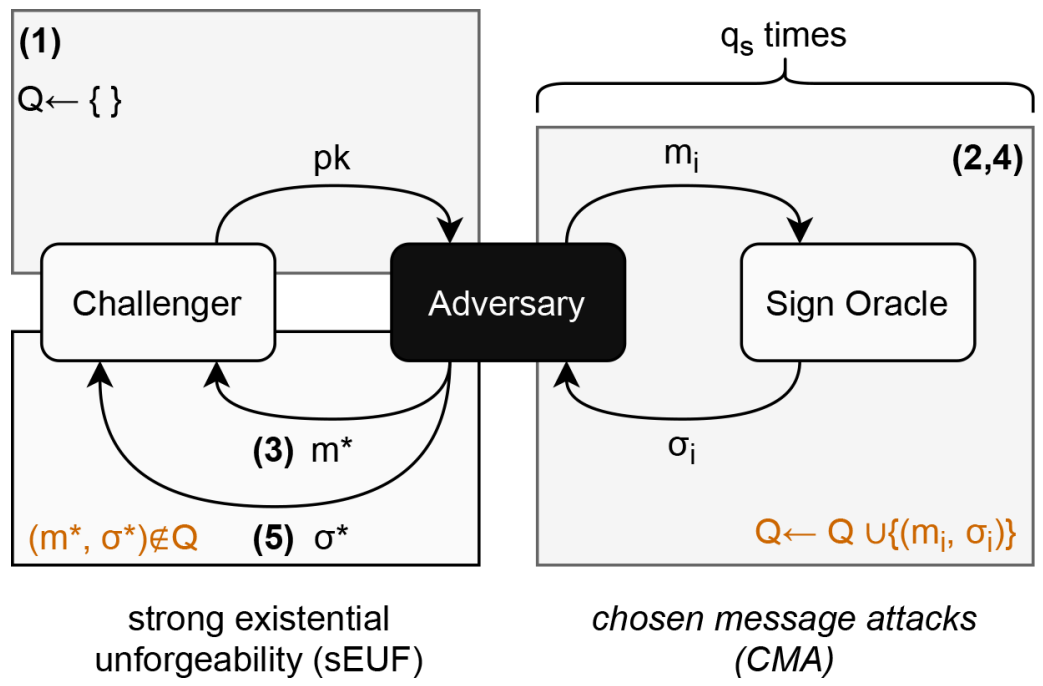

Figure 4. SEUF-CMA security model.

$\sigma^{*}$. We say the adversary breaks the DSS if $\sigma^{*}$ on $m^{*}$ is valid. $q_{s}$ quantifies the number message-signature pair made available to the adversary.

\section{Strong existential unforgeability (sEUF-CMA)}

Consider the case for randomized signatures in the EUF-CMA model (e.g., DSA ${ }^{13}$ or PSS- ${ }^{18}$ ). A randomized DSS allows multiple valid signatures for a single message, which has a subtle implication on the model: Suppose that the adversary queried for a message-signature pair $(m, \sigma)$. Now, the adversary forges a different valid signature $\sigma^{\prime} \neq \sigma$ on the same message $m$. This is an easier security goal, but could be a critical vulnerability when the DSS is used in a scenario in which the designers assumed that no new signatures can be forged, because a different signature on an existing message is still new. In other words, EUF-CMA does not guarantee that if that an attacker knows $(m, \sigma)$, it cannot forge $\left(m, \sigma^{\prime}\right)$ such that $\sigma^{\prime}$ on $m$ is valid. This gave rise to a stronger security model, known as strong existential unforgeability or sEUFCMA. ${ }^{25}$ Figure 4 shows the interactions of the adversary with the challenger in the sEUF-CMA model. The main difference is the constraint during the chosen message attacks and in the final step (5). Notably, the adversary can even query for signatures on the challenge message $m^{*}$, but may not submit any of the signatures obtained from the sign oracle as forgeries. In contrast to EUF-CMA, sEUF-CMA ensures that an adversary cannot produce any new signatures at all; any valid signature must have originated from the signer.

\section{Why does sEUF-CMA matter?}

Let's take a step back and consider why sEUF-CMA is even worth considering in the first place. DSS is very often used as a building block to construct other cryptographic schemes. For example, using the Fiat-Shamir transform, ${ }^{26}$ a DSS can be turned into an identification protocol. DSS has also been used to create identity-based identification schemes, ${ }^{27}$ signcryption schemes, ${ }^{25}$ authenticated key exchanges, ${ }^{28}$ and identity-based encryption schemes. ${ }^{29}$ DSS with EUF-CMA security is found to be insufficient in some of the constructions, particularly to build non-malleable cryptographic schemes. In a nutshell, non-malleability refers to the impossibility of an adversary to generate a different ciphertext to some previously known ciphertext that decrypts to the same message, which is a desirable property in cryptographic schemes. ${ }^{30}$ Thus, if a DSS is sEUF-CMA, it is much more versatile because it can be useful as a building block for many of the schemes which requires the property of non-malleability.

\section{A simple attack for when a DSS is not sEUF-CMA}

We show a toy example of a simple attack that can be achieved by an attacker if the DSS used for authentication is not sEUF-CMA. Figure 5 shows honest users Alice and Bob, as well as an attacker Mallory which has hijacked the channel. In step (1), Bob wants to authenticate that Alice is truly on the other end. Mallory launches a chosen message attack in step (2) and (3) on Alice and obtains the message $m$ and signature $\sigma$. In step (4), Mallory forwards $(m, \sigma)$ to Bob, which may initially convince them. After some time elapsed, Bob wants to re-authenticate Alice to ensure that they are still who they claim to be (5). Mallory forges a valid signature $\sigma^{\prime} \neq \sigma$ on $m$ and forwards that to Bob (6). From Bob's perspective, Alice generating a different signature ought to warrant some confidence that Alice is truly Alice. However, if the DSS is not sEUF, this is not the case, as Bob is obviously duped. 


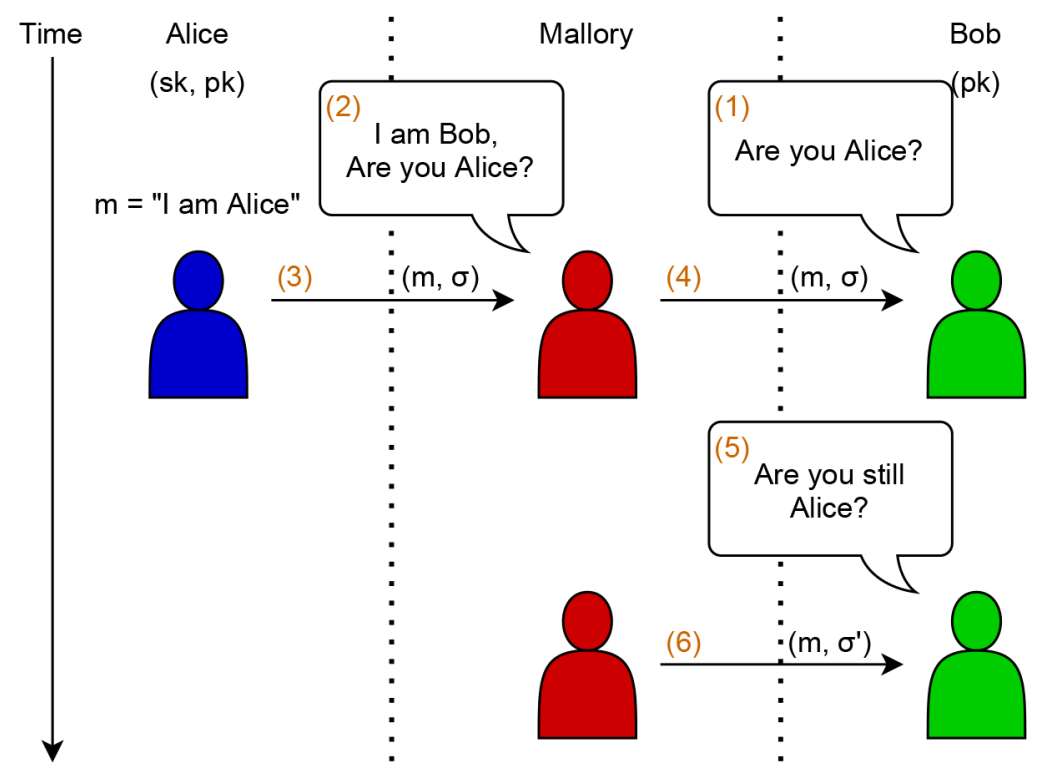

Figure 5. A simple problem on a digital signature schemes without sEUF-CMA.

\section{Real life problems arising from signature malleability (non-sEUF)}

We established sEUF-CMA is of theoretical interest. Here we briefly mention a few real-life needs for sEUF-CMA to emphasize the importance of it. Not too long ago, Stern et al. showed how to duplicate ECDSA signatures based on their malleability. ${ }^{31}$ A more recent example is the transaction malleability of the popular cryptocurrency, Bitcoin. When a transaction in Bitcoin is signed, the signature does not cover the entire transaction used for hashing; an attacker could perform strong forgery (i.e., maul the signature), then claim that the transaction has failed (because the hash is not valid). The sender would believe it because the signature is valid! This leads to the sender issuing a new transaction, without knowing that the original transaction is valid. ${ }^{32,33}$ Decker and Wattenhofer pointed out that this subtle flaw could be responsible for a small portion of Bitcoins stolen during the $2014 \mathrm{MtGox}$ attack. ${ }^{34}$ They remarked that transaction malleability should be carefully considered when implementing Bitcoin clients. In another research, Jackson et al. found that signatures that are not sEUF-CMA may cause the strong session agreement of a well-known authenticated key exchange algorithm, the STS-ISO ${ }^{1}$ to fail. ${ }^{36}$ They analyzed STS-ISO using the Tamarin Prover and found that if sEUF is violated, attackers can force the parties in the session to accept message that do not originate from either of the honest parties.

\section{DSS secure in SEUF-CMA}

The notion of sEUF-CMA first appeared in the form of non-malleability of ciphertexts in the works of Dolev, Dwork and Naor $^{30}$ in 1991. The term sEUF-CMA was first introduced by An, Dodis and Rabin which presented it as requirement to construct signcryption schemes. ${ }^{25}$ Boneh, Shen and Waters were the first DSS that is considered and proven in the sEUFCMA model,${ }^{37}$ but mentions that DSS that are sEUF-CMA have existed earlier such as full domain hash (FDH)-RSA. ${ }^{18}$ FDH-RSA must be sEUF-CMA because the signatures generated are determined exactly by the message only. This means that FDH-RSA signatures are deterministic. For deterministic signatures, a strong forgery cannot occur without the message $m^{\prime}$ being different from the original $m$, therefore, deterministic signatures are generally sEUF-CMA. We see that this is also the case for the widely used and popular EdDSA which had recently received a provable security treatment. ${ }^{38}$ Boneh, Shen and Waters shows that non-deterministic signatures may also exhibit sEUF-CMA, such as the Micali-Reyzin signatures, ${ }^{39}$ Goh-Jarecki signatures ${ }^{40}$ and Boneh-Boyen signatures. ${ }^{11}$ The reason for this is that if the forger manages to re-randomize a signature on the same message, the signature constitutes an existential forgery through clever binding of the messages. This is a recurring paradigm to design sEUF-CMA signature schemes. ${ }^{11,41}$ Since the introduction of the sEUF-CMA model, existing EUF-CMA secure DSS are now re-considered in the sEUF-CMA model. An example of such work is by Fersch, Kiltz and Poettering on the well established DSA. ${ }^{13}$ DSA was shown to be secure under sEUF-CMA, while ECDSA was not ${ }^{2}{ }^{43}$ More recently in 2021, Brendel et al. proved the IETF version of EdDSA to be sEUF-CMA secure. ${ }^{38}$ Table 1 shows a list of DSS that are secure under sEUF-CMA.

${ }^{1}$ STS stands for Station-to-Station and is an authenticated key exchange protocol due to Diffie et al. ${ }^{35}$

${ }^{2}$ Brown proved ECDSA to secure under sEUF-CMA, ${ }^{42}$ but this was refuted through a series of trivial attacks posed by. ${ }^{43}$ 


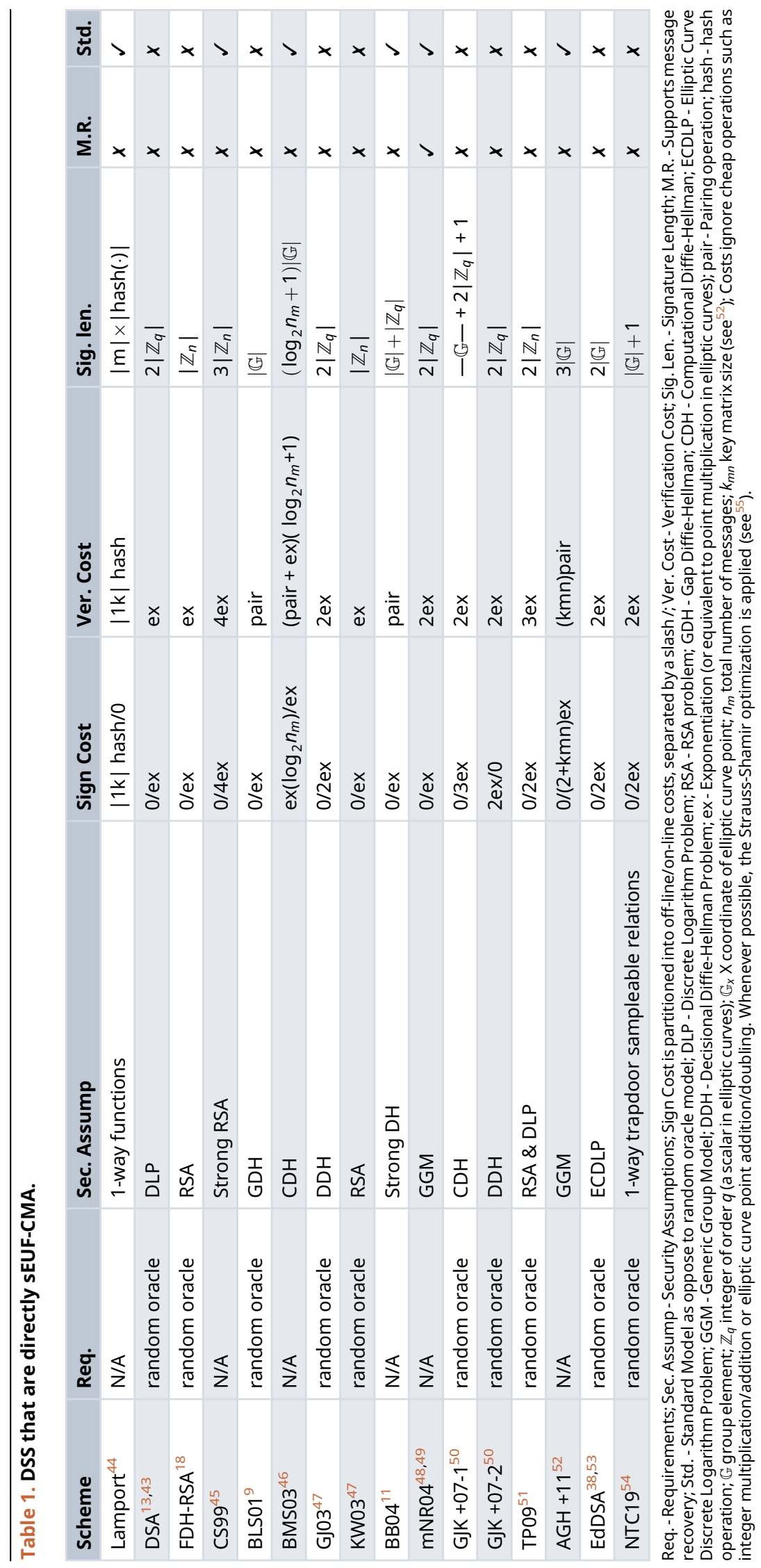




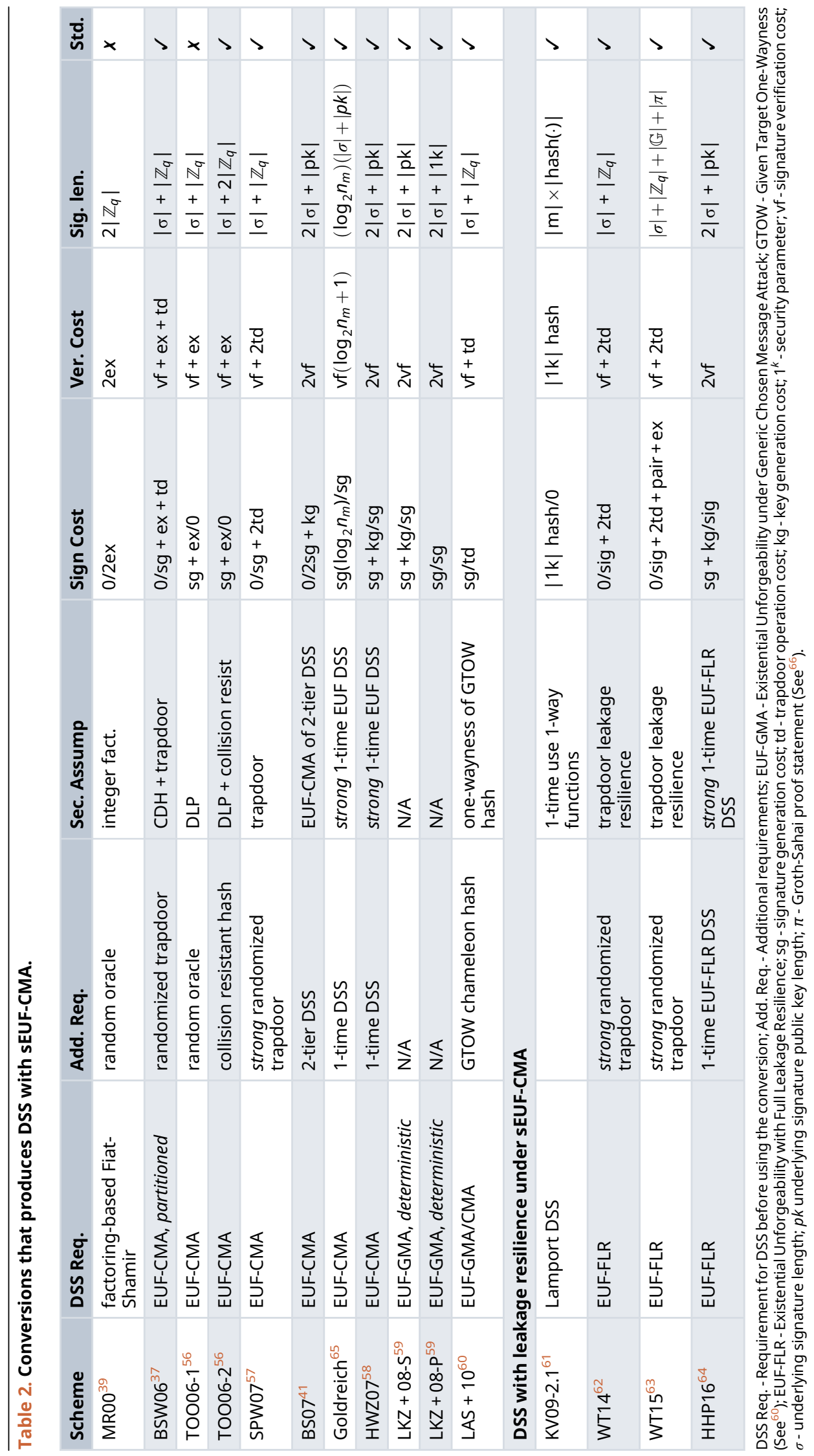




\section{Message recovery}

From another perspective, we consider DSS with message recovery (DSS-R) candidates under sEUF-CMA. As pointed out by Ateniese and de Mediros, the modified Nyberg-Rueppel signature is sEUF-CMA secure. ${ }^{49}$ While it is tempting to think that DSS-R must be sEUF-CMA, because modifying the signature will surely modify the message itself given that one can recover the message from the signature. However, we see that this is not the case as Ateniese and de Mediros found the original Nyberg-Rueppel signatures ${ }^{49}$ to be insecure in sEUF-CMA.

\section{Conversions to sEUF-CMA}

Boneh, Shen and Waters opened a new field in the research of DSS under sEUF-CMA: Interest in sEUF-CMA conversion starts to accumulate with various works being published. ${ }^{41,56-58}$ Instead of working on individual DSS, the line of research focuses on creating efficient conversions to enhance existing generic DSS with EUF-CMA into sEUF-CMA. Some transforms do not even need EUF-CMA security at minimum, only requiring EUF-GMA, which is a weaker notion than EUF-CMA. ${ }^{59,60}$ Table 2 shows a list of conversion methods since 2006, including the use of DSS in leakage resilient settings. $^{61-64}$

\section{Widely used DSS that have malleable signatures}

Through our research, we investigated some of the most used DSS in the industry on their security in the sEUF-CMA model. The following are the popular DSS that are not sEUF-CMA secure.

\section{RSA PKCS\#1.5}

\section{2. $\operatorname{ECDSA}^{31,43}$}

3. Ed25519 (Original, not IETF RFC 8032) 38

\section{Challenges and future direction}

In recent work, there has been several post-quantum cryptographic DSS that incorporates the sEUF-CMA model during design. ${ }^{68-71}$ DSS in various other contexts (e.g., privacy preserving computation, multiparty computation) such as a homomorphic DSS, ${ }^{72}$ group DSS, ${ }^{73}$ and proxy DSS ${ }^{74-76}$ are being considered in sEUF-CMA as well. In addition, DSS in even more complex cryptographic settings such as in certificateless and identity-based settings are also using sEUF-CMA as their standard model for security. ${ }^{77-80}$ We see that the security goal post has been moved from EUF-CMA to sEUFCMA in the span of a decade and believe this is the right direction forward as DSS is increasingly used in intricate security protocols, which cannot tolerate any design flaw that arises from as simple as malleable signatures.

\section{Conclusion}

In this work, we provided a comprehensive review on what is strong unforgeability in DSS, why is it needed, which of the DSS are secure under the model and how to obtain it if the DSS is only existentially unforgeable. We surveyed and analyzed existing DSS in literature which are secure under sEUF-CMA, and noted the requirements, computational and storage efficiency as well as the security assumptions of each DSS to provide an overview of DSS under the much more secure model.

\section{Data availability}

No data is associated with this article.

\section{Acknowledgements}

We would like to acknowledge our research funder: This research is supported by the Ministry of Higher Education of Malaysia through the Fundamental Research Grant Scheme under Grant FRGS/1/2019/ICT04/MMU/02/5 and in part by Multimedia University's Research Management Fund.

References

1. Diffie W, Hellman M: New directions in cryptography. IEEE Trans. Inf. Theor. September 2006; 22(6): 644-654. 0018-9448. Publisher Full Text
2. Rivest RL, Shamir A, Adleman L: A method for obtaining digital signatures and public-key cryptosystems. Commun. ACM February 1978; 21(2): 120-126. 0001-0782. Publisher Full Text 
3. Katz J, Lindell Y: Introduction to Modern Cryptography (Chapman \& Hall/Crc Cryptography and Network Security Series). Chapman \& Hall/ CRC; 2007. 1584885513.

4. Kaliski B: PKCS \#1: RSA Encryption Version 1.5. RFC 2313, March

Reference Source

5. Bellare M, Rogaway P: Random oracles are practical: A paradigm for designing efficient protocols. Proc 1st ACM Conf Computer Communications Security., CCS '93, New York, NY, USA: Association for Computing Machinery; 1993; page 62-73. 0897916298. Publisher Full Text

6. Gennaro R, Halevi S, Rabin T: Secure hash-and-sign signatures without the random oracle. In: Stern J, editor Advances in Cryptology - EUROCRYPT '99. Berlin, Heidelberg: Springer Berlin Heidelberg; 1999; pages 123-139. 978-3-540-48910-8.

7. Cramer R, Shoup V: Signature schemes based on the strong rsa assumption. ACM Trans. Inf. Syst. Secur. August 2000; 3(3): 161-185. 1094-9224.

Publisher Full Text

8. Kaliski B, Staddon J: PKCS \#1: RSA Cryptography Specifications Version 2.0. RFC 2437. October 1998

Reference Source

9. Boneh $D$, Lynn B, Shacham H: Short signatures from the weil pairing. In: Boyd C, editor, Advances in Cryptology - ASIACRYPT 2001. Berlin, Heidelberg: Springer Berlin Heidelberg; 2001; pages 514-532. 978-3-540-45682-7.

10. Waters BR: Efficient identity-based encryption without random oracles. Cryptology ePrint Archive, Report 2004/180. 2004. Reference Source

11. Boneh $D$, Boyen $X$ : Short signatures without random oracles. In: Cachin C, Camenisch JL, editors, Advances in Cryptology - EUROCRYPT 2004. Berlin, Heidelberg: Springer Berlin Heidelberg; 2004; pages 56-73. 978-3-540-24676-3.

12. Johnson $D$, Menezes $A$, Vanstone $S$ : The elliptic curve digital signature algorithm (ECDSA). Int. J. Inf. Secur. August 2001; 1(1): 36-63. 1615-5262.

Publisher Full Text

13. National Institute of Standards and Technology: Digital signature standard (DSS). Technical Report Federal Information Processing Standards Publications (FIPS PUBS) 186-4, Change Notice 4July 19, 2013, U.S. Washington, D.C.: Department of Commerce; 2013.

14. Hofheinz D, Jager T, Kiltz E: Short signatures from weaker assumptions. In: Lee Dong Hoon, Wang X, editors, Advances in Cryptology - ASIACRYPT. Berlin, Heidelberg: Springer Berlin Heidelberg; 2011, pages 647-666. 978-3-642-25385-0.

15. Seo JH: Efficient digital signatures from rsa without random oracles. Info Sci. 2020; 512: 471-480. 0020-0255. Publisher Full Text|Reference Source

16. Nyberg K, Rueppel Rainer A: Message recovery for signature schemes based on the discrete logarithm problem. In: $\mathrm{De}$ Santis A, editor, Advances in Cryptology - EUROCRYPT'94. Berlin, Heidelberg: Springer Berlin Heidelberg; 1995; pages 182-193. 9783-540-44717-7.

17. Miyaji A: A message recovery signature scheme equivalent to dsa over elliptic curves. 11 1996; 1163. Publisher Full Text

18. Bellare $M$, Rogaway $P$ : The exact security of digital signatureshow to sign with rsa and rabin. In: Maurer $U$, editor, Advances in Cryptology - EUROCRYPT '96. Berlin, Heidelberg: Springer Berlin Heidelberg; 1996; pages 399-416. 978-3-540-68339-1.

19. Abe $M, O$ okamoto $T$ : A signature scheme with message recovery as secure as discrete logarithm. In: Lam Kwok-Yan, Okamoto $E$, Xing C, editors, Advances in Cryptology - ASIACRYPT'99. Berlin, Heidelberg: Springer Berlin Heidelberg; 1999; pages 378-389. 9783-540-48000-6.

20. Tzeng Shiang-Feng, Hwang Min-Shiang: Digital signature with message recovery and its variants based on elliptic curve discrete logarithm problem. Computer Standards \& Interfaces. 2004; 26(2): 61-71. 0920-5489.

Publisher Full Text | Reference Source

21. Sekhar MR: Signature scheme with message recovery and its application. Int J Computer Mathematics. 2004; 81(3): 285-289. Publisher Full Text

22. Tahat $\mathrm{N}$, Shaqboua $\mathrm{R}$, Abdallah $\mathrm{E}$, et al.: A new digital signature scheme with message recovery using hybrid problems. Int J Electrical Computer Eng (IJECE). 10 2019; 9: 3576. Publisher Full Text

23. Kazmirchuk S, Ilyenko A, Ilyenko S: Digital Signature Authentication Scheme with Message Recovery Based on the Use of Elliptic Curves. 01 2020; pages 279-288. 978-3-030-16620-5.

Publisher Full Text
24. Goldwasser S, Micali S, Rivest Ronald L: A digital signature scheme secure against adaptive chosen-message attacks. SIAM J. Comput. April 1988; 17(2): 281-308. 0097-5397. Publisher Full Text

25. An JH, Dodis $Y$, Rabin T: On the security of joint signature and encryption. In: Knudsen LR, editor, Advances in Cryptology EUROCRYPT 2002. Berlin, Heidelberg: Springer Berlin Heidelberg; 2002; pages 83-107. 978-3-540-46035-0.

26. Fiat A, Shamir A: How to prove yourself: Practical solutions to identification and signature problems. In: Odlyzko Andrew $M$, editor, Advances in Cryptology - CRYPTO' 86. Berlin, Heidelberg: Springer Berlin Heidelberg; 1987; pages 186-194. 978-3-54047721-1.

27. Kurosawa $\mathrm{K}$, Heng S-H: From digital signature to id-based identification/signature. In: Bao F, Deng R, Zhou J, editors, Public Key Cryptography - PKC 2004. Berlin, Heidelberg: Springer Berlin Heidelberg; 2004; pages 248-261. 978-3-540-24632-9.

28. Katz J, Yung M: Scalable protocols for authenticated group key exchange. In: Boneh D, editor, Advances in Cryptology-CRYPTO 2003. Berlin, Heidelberg: Springer Berlin Heidelberg; 2003; pages 110-125. 978-3-540-45146-4.

29. Canetti R, Halevi S, Katz J: Chosen-ciphertext security from identity-based encryption. In: Cachin C, Camenisch JL, editors, Advances in Cryptology - EUROCRYPT 2004. Berlin, Heidelberg: Springer Berlin Heidelberg; 2004; pages 207-222. 978-3-540 24676-3.

30. Dolev D, Dwork C, Naor M: Non-malleable cryptography. SIAM J Computing. 03 1991; 30. Publisher Full Text

31. Stern J, Pointcheval D, Malone-Lee J, et al.: Flaws in applying proof methodologies to signature schemes. In: Yung M, editor, Advances in Cryptology - CRYPTO 2002. Berlin, Heidelberg: Springer Berlin Heidelberg; 2002; pages 93-110. 978-3-540-45708-4.

32. Bitcoin transaction malleability: 2017. Reference Source Accessed: 2021-06-03.

33. Transaction malleability: Bitcoin Wiki [Online]. 2018. Accessed: 2021-06-03.

Reference Source

34. Decker C, Wattenhofer R: Bitcoin transaction malleability and mtgox. Lecture Notes in Computer Science. 2014; page 313-326. 1611-3349.

Publisher Full Text

35. Diffie W, Van Oorschot PC, Wiener MJ: Authentication and authenticated key exchanges. Des. Codes Cryptography. June 1992; 2(2): 107-125. 0925-1022. Publisher Full Text

36. Jackson D, Cremers C, Cohn-Gordon K, et al.: Seems legit: Automated analysis of subtle attacks on protocols that use signatures. In Proc of the 2019 ACM SIGSAC Conference on Computer and Communications Security, CCS '19. New York, NY, USA Association for Computing Machinery; 2019; page 2165-2180. 9781450367479 Publisher Full Text

37. Boneh $D$, Shen $E$, Waters $B$ : Strongly unforgeable signatures based on computational diffie-hellman. In: Yung $M$, Dodis $Y$, Kiayias A, et al., editors, Public Key Cryptography - PKC 2006. Berlin, Heidelberg: Springer Berlin Heidelberg; 2006; pages 229-240. 9783-540-33852-9.

38. Brendel J, Cremers C, Jackson D, et al.: The provable security of ed25519: Theory and practice. 20212021 IEEE Symposium on Security and Privacy (SP). Los Alamitos, CA, USA: IEEE Computer Society; may 2021; pages 715-732.

Publisher Full Text | Reference Source

39. Micali S, Reyzin L: Improving the exact security of digital signature schemes. J. Cryptology. 03 2002; 15: 1-18. Publisher Full Text

40. Goh Eu-lin, Jarecki Stanisław: A signature scheme as secure as the diffie-hellman problem. In: Biham E, editor, Advances in Cryptology - EUROCRYPT 2003. Berlin, Heidelberg: Springer Berlin Heidelberg; 2003; pages 401-415. 978-3-540-39200-2.

41. Bellare M, Shoup S: Two-tier signatures, strongly unforgeable signatures, and fiat-shamir without random oracles. In: Okamoto T, Wang X, editors, Public Key Cryptography - PKC 2007. Berlin, Heidelberg: Springer Berlin Heidelberg; 2007; pages 201-216. 978-3-540-71677-8.

42. Brown D: On the Provable Security of ECDSA, page 21-40. London Mathematical Society Lecture Note Series: Cambridge University Press; 2005

Publisher Full Text

43. Fersch M, Kiltz E, Poettering B: On the provable security of (ec)dsa signatures. Proc 2016 ACM SIGSAC Conference on Computer and Communications Security, CCS '16. New York, NY, USA: Association for 
Computing Machinery; 2016; page 1651-1662. 9781450341394. Publisher Full Text

44. Lamport $\mathrm{L}$ : Constructing digital signatures from a one way function. Technical Report CSL-98. October 1979. Reference Source This paper was published by IEEE in the Proceedings of HICSS-43 in January, 2010.

45. Cramer R, Shoup V: Signature schemes based on the strong rsa assumption. Proc of the 6th ACM Conference on Computer and Communications Security, CCS '99. New York, NY, USA: Association for Computing Machinery; 1999; page 46-51. 1581131488 Publisher Full Text

46. Boneh D, Mironov I, Shoup V: A secure signature scheme from bilinear maps. In: Joye M, editor, Topics in Cryptology - CT-RSA 2003. Berlin, Heidelberg: Springer Berlin Heidelberg; 2003; pages 98-110. 978-3-540-36563-1.

47. Katz J, Wang N: Efficiency improvements for signature schemes with tight security reductions. Proceedings of the 10th ACM Conference on Computer and Communications Security, CCS '03. New York, NY, USA: Association for Computing Machinery; 2003; page 155-164. 1581137389

Publisher Full Text

48. Ateniese $\mathrm{G}$, de Medeiros $\mathrm{B}$ : Efficient group signatures without trapdoors. In: Laih C-S, editor, Advances in Cryptology - ASIACRYPT 2003. Berlin, Heidelberg: Springer Berlin Heidelberg; 2003; pages 246-268. 978-3-540-40061-5.

49. Ateniese G, Medeiros BD: A provably secure nyberg-rueppel signature variant with applications. IACR Cryptol. ePrint Arch. 2004:93, 2004

50. Goh E-J, Jarecki S, Katz J, et al.: Efficient signature schemes with tight reductions to the diffie-hellman problems. J. Cryptol. 2007; 20 (4): 493-514.

Publisher Full Text

51. Tantikittipisut W, Premasathian N: Strongly unforgeable signature scheme without one-time key. 2009 Eighth IEEE/ACIS Int Conf Computer Information Sci. 2009; pages 313-316. Publisher Full Text

52. Abe M, Groth J, Haralambiev K, et al.: Optimal structure-preserving signatures in asymmetric bilinear groups. CRYPTO. 2011. Publisher Full Text

53. Josefsson S, Liusvaara I: Edwards-Curve Digital Signature Algorithm (EdDSA). RFC 8032. January 2017.

Reference Source

54. Ng T-S, Tan S-Y, Chin J-J: Improving signature schemes with tight security reductions. In: Heng S-H, Lopez J: editors, Information Security Practice and Experience. Cham: Springer International Publishing; 2019; pages 273-292. ISBN 978-3-030-34339-2.

55. Rondepierre F: Revisiting atomic patterns for scalar multiplications on elliptic curves. Cryptology ePrint Archive, Report 2015/408. 2015.

Reference Source

56. Teranishi I, Oyama T, Ogata W: General conversion for obtaining strongly existentially unforgeable signatures. In: Barua $\mathrm{R}$, Lange T: editors, Progress in Cryptology - INDOCRYPT 2006. Berlin, Heidelberg: Springer Berlin Heidelberg; 2006; pages 191-205. 978-3-540-49769-1.

57. Steinfeld R, Pieprzyk J, Wang H: How to strengthen any weakly unforgeable signature into a strongly unforgeable signature. In: Abe M: editor, Topics in Cryptology - CT-RSA 2007. Berlin, Heidelberg: Springer Berlin Heidelberg; 2006; pages 357-371. 978-3-540-69328-4.

58. Huang $Q$, Wong DS, Zhao Y: Generic transformation to strongly unforgeable signatures. In: Katz J, Yung M: editors, Applied Cryptography and Network Security. Berlin, Heidelberg: Springer Berlin Heidelberg; 2007; pages 1-17. 978-3-540-72738-5.

59. Li J, Kim K, Zhang F, et al.: Generic security-amplifying methods of ordinary digital signatures. In: Bellovin SM, Gennaro R, Keromytis A, et al.: editors, Applied Cryptography and Network Security. Berlin, Heidelberg: Springer Berlin Heidelberg; 2008; pages 224-241. 978-3-540-68914-0.

60. Liu JK, Au MH, Susilo W, et al.: Short generic transformation to strongly unforgeable signature in the standard model. In: Gritzalis D, Preneel B, Theoharidou M: editors, Computer Security ESORICS 2010. Berlin, Heidelberg: Springer Berlin Heidelberg; 2010; pages 168-181. 978-3-642-15497-3.

61. Katz J, Vaikuntanathan V: Signature schemes with bounded leakage resilience. In: Matsui M: editor, Advances in Cryptology ASIACRYPT 2009. Berlin, Heidelberg: Springer Berlin Heidelberg; 2009; pages 703-720. 978-3-642-10366-7.

62. Wang $Y$, Tanaka K: Generic transformation to strongly existentially unforgeable signature schemes with leakage resiliency. In: Chow SSM, Liu JK, Hui LCK, et al.: editors, Provable Security. Cham: Springer International Publishing; 2014; pages 117-129. 978-3-319-12475-9.

63. Wang $Y$, Tanaka K: Generic transformation to strongly existentially unforgeable signature schemes with continuous leakage resiliency. In: Foo E, Stebila D: editors, Information Security and Privacy. Cham: Springer International Publishing; 2015; pages 213-229. 978-3-319-19962-7.

64. Huang J, Huang Q, Pan C: A black-box construction of strongly unforgeable signature schemes in the bounded leakage model. In: Chen L, Han J: editors, Provable Security. Cham: Springer International Publishing; 2016; pages 320-339. 978-3-319-47422-9.

65. Goldreich O: The foundations of cryptography - volume 2: Basic applications. 2001

66. Groth J, Sahai A: Efficient non-interactive proof systems for bilinear groups. Cryptology ePrint Archive, Report 2007/155. 2007. Reference Source

67. Moriarty K, Kaliski B, Jonsson J, et al.: PKCS \#1: RSA Cryptography Specifications Version 2.2. RFC 8017. November 2016. Reference Source Section 9.2, Note 2 .

68. Rückert M: Strongly unforgeable signatures and hierarchical identity-based signatures from lattices without random oracles. In: Sendrier N: editor, Post-Quantum Cryptography. Berlin, Heidelberg: Springer Berlin Heidelberg; 2010; pages 182-200. 9783-642-12929-2.

69. Noh G, Chun JY, Jeong IR: Strongly unforgeable ring signature scheme from lattices in the standard model. J Appl Mathematics, 2014. 2014. 1110-757X Publisher Full Text

70. Eaton E, Song F: Making existential-unforgeable signatures strongly unforgeable in the quantum random-oracle model. arXiv preprint arXiv:1509.02944. 2015

71. Cremers C, Düzlü S, Fiedler R, et al.: Buffing signature schemes beyond unforgeability and the case of post-quantum signatures. Cryptology ePrint Archive, Report 2020/1525. 2020 Reference Source

72. Wang $\mathrm{F}$, Wang $\mathrm{K}$, Bao $\mathrm{L}$, et al.: Leveled strongly-unforgeable identity-based fully homomorphic signatures. In: López J, Mitchell CJ: editors, Information Security - 18th International Conference, ISC 2015, Trondheim, Norway, September 9-11, 2015, Proceedings volume 9290 of Lecture Notes in Computer Science. Springer; 2015; pages 42-60. Publisher Full Text

73. Park H, Lim S, Yie I, et al.: Strong unforgeability in group signature schemes. Computer Standards Interfaces. 2009; 31(4): 856-862. 0920 5489.

Publisher Full Text | Reference Source

74. Sun $Y, X u C, Y u Y$, et al.: Strongly unforgeable proxy signature scheme secure in the standard model. J Syst Soft. 2011; 84(9): 1471-1479. 0164-1212.

Publisher Full Text | Reference Source Selected papers from the 2009 Joint Working IEEE/IFIP Conference on Software Architecture \& European Conference on Software Architecture (WICSA/ECSA 2009).

75. Yu Y, Yi M, Susilo W, et al.: Provably secure proxy signature scheme from factorization. Mathematical and Computer Modelling. 2012; 55(3): 1160-1168, 0895-7177. Publisher Full Text | Reference Source

76. Pang $L$, Zhao $H$, Zhou $X$, et al.: Strongly unforgeable and efficient proxy signature scheme with fast revocation secure in the standard model. Int J Distributed Sensor Networks. 2016; 12 (1): 3205206. Publisher Full Text

77. Sato C, Okamoto T, Okamoto E: Strongly unforgeable id-based signatures without random oracles. In: Bao F, Li H, Wang G: editors, Information Security Practice and Experience. Berlin, Heidelberg: Springer Berlin Heidelberg; 2009: pages 35-46. 978-3642-00843-6.

78. Zhang J, Liu X: An efficient strong id-based signature scheme with unforgeability. 2010 Fifth International Conference on Frontier of Computer Science and Technology. 2010; pages 239-245. Publisher Full Text

79. Yu Y, Yi M, Wang G, et al.: Improved certificateless signature scheme provably secure in the standard model. Information Security, IET. 06 2012; 6: 102-110. Publisher Full Text

80. Liu Z, Zhang X, Hu Y, et al.: Revocable and strongly unforgeable identity-based signature scheme in the standard model. Sec. and Commun. Netw. September 2016; 9(14): 2422-2433. 1939-0114.

Publisher Full Text 


\section{Open Peer Review}

\section{Current Peer Review Status:}

\section{Version 1}

Reviewer Report 13 February 2023

https://doi.org/10.5256/f1000research.76522.r162696

(C) 2023 Teh P. This is an open access peer review report distributed under the terms of the Creative Commons Attribution License, which permits unrestricted use, distribution, and reproduction in any medium, provided the original work is properly cited.

\section{Pin Shen Teh}

Manchester Metropolitan University, Manchester, UK

The research objective was clear.

The concept of the work was straightforward.

The presentation style of the article was clear.

Grammar mistakes are noticeable. The sentence structure and choice of words used can be more concise and precise, some terms used were somewhat inconsistent, making reading and understanding the article quite challenging.

Further elaboration of why the three popular DSS that are not SEUF-CMA secure would be helpful.

Overall the depth of the work is sufficient for the calibre of this journal.

Is the topic of the review discussed comprehensively in the context of the current literature?

Yes

Are all factual statements correct and adequately supported by citations?

Yes

Is the review written in accessible language?

Yes

Are the conclusions drawn appropriate in the context of the current research literature? Yes 
Competing Interests: No competing interests were disclosed.

Reviewer Expertise: Biometrics, Security, Machine Learning, Mobile Authentication

I confirm that I have read this submission and believe that I have an appropriate level of expertise to confirm that it is of an acceptable scientific standard.

Reviewer Report 03 November 2021

https://doi.org/10.5256/f1000research.76522.r97082

(C) 2021 Wong D. This is an open access peer review report distributed under the terms of the Creative Commons Attribution License, which permits unrestricted use, distribution, and reproduction in any medium, provided the original work is properly cited.

\section{Denis Chee-Keong Wong}

Lee Kong Chian Faculty of Engineering and Science, Universiti Tunku Abdul Rahman, Kampar, Malaysia

The paper performs a review on existing DSS together with its properties, such as public verifiability, non-repudiation, and ability for message recovering. Furthermore, a detailed discussion on DSS security models are included, which are the EUF-CMA and SEUF-CMA. The differences between both security models are elaborated upon in detail. Finally, the authors provided comprehensive information on existing DSS in Table 1 for comparison purposes. As a survey paper, this work is comprehensive and suitable to be indexed. The authors need to recheck some of the entries in Table 1 to ensure the information stated is correct - please look at the "GJK +07-1", and please ensure the group G used is the same for all schemes with $|G|$ in the column of "sig. len.".

Is the topic of the review discussed comprehensively in the context of the current literature?

Yes

Are all factual statements correct and adequately supported by citations?

Yes

Is the review written in accessible language?

Yes

Are the conclusions drawn appropriate in the context of the current research literature? Yes

Competing Interests: No competing interests were disclosed.

Reviewer Expertise: Algebraic cryptography, algebra 
I confirm that I have read this submission and believe that I have an appropriate level of expertise to confirm that it is of an acceptable scientific standard.

The benefits of publishing with F1000Research:

- Your article is published within days, with no editorial bias

- You can publish traditional articles, null/negative results, case reports, data notes and more

- The peer review process is transparent and collaborative

- Your article is indexed in PubMed after passing peer review

- Dedicated customer support at every stage

For pre-submission enquiries, contact research@f1000.com 